\title{
New Apparatus Stimulating the Region of Tongue Innervated by Glossopharyngeal Nerve and Its Application to Monosodium Glutamate (MSG) Solution
}

\author{
Byung Chan Min ${ }^{11}$, Masashi Wada2) and Kazuyoshi Sakamoto1)
}

1) Ergonomics Lab., Department of Communi cations and Systems, The University of Electro-Communications

2) Department of Otorhinolaryngology, National Center of Neurology and Psychiatry, Kohnodai Hospital

\begin{abstract}
A bstract. Some apparatuses stimulating the region of tongue innervated by chorda tympani nerve were well known and they were used popularly, but the apparatuses for glossopharyngeal nerve were not produced yet. In the study, the apparatus for the stimulations of glossopharyngeal nerve was trially produced. It was known that the substance of "Umami" was responded well in the tongue region innervated by glossopharyngeal nerve. The evoked potential for the stimulations of monosodium glutamate (MSG) solution which was known as substance of Umami was measured with the use of the trial stimulating apparatus. The evoked potential was detected by monopolar lead for the positions between $\mathrm{Cz}$ and $\mathrm{Al}$ (left lobe) with the ground at F pz. The evoked potential of MSG consisted of a positive peak wave with the latency at approximately $250 \mathrm{~ms}$. The amplitudes increased and the latency decreased as the concentration of MSG increased. The evoked potential stimulating the tongue innervated by this glossopharyngeal nerve could be detected for the first time by the use of the trial apparatus.
\end{abstract}

(Appl Human Sci, 17 (2): 67-71, 1998)

Keywords: gustatory evoked potentials, glossopharyngeal nerve, monosodium glutamate, MSG, Umami

\section{Introduction}

Current status of gustatory evoked potentials

Visual, auditory and somatosensory evoked potentials have been proven to be useful for noninvasive assessment of neurophysiological function in normal and clinical populations (Chiappa, 1983). Recording of gustatory evoked potential (GEP) as a method for objective evaluation of tastes was first attempted by Funakoshi and Kawamura (1968 and 1971), followed by Kobal (1985), Ikui (1988), Maetani et al. (1989), Platting (1991), and Min and Sakamoto (1997 and 1998), but a clinically applicable method has not yet been established. Funakoshi and Kawamura (1968 and 1971) reported that the latencies of evoked potentials obtained spreaded over the wide range of 500-1500 ms. Kobal (1985) developed the apparatus for gaseous taste stimulus to remove touch stimuli, and he obtained pure taste stimulus. He measured the evoked potentials using the stimulus with the rising time below 20 $\mathrm{ms}$ and the duration of $200 \mathrm{~ms}$. The obtained latencies were composed of four major components; that is, the positive peak at $300 \mathrm{~ms}$, the negative peak at $410 \mathrm{~ms}$, the next positive peak at $660 \mathrm{~ms}$, and the next negative peak at $860 \mathrm{~ms}$. Although definite evoked potentials were obtained by gaseous taste stimulus, the stimulus did not make clear the definite number of taste organs stimulated or the definite innervation area of taste organ activated by the gaseous stimulus. Ikui (1988), using sophisticated gustatory stimulation that delivered taste solution to the small area of the tongue surface innervated by the chorda tympani nerve, described a negative evoked potentials (200 600 ms) produced by solutions of $1 \mathrm{M} \mathrm{NaCl}$. Maetani et al. (1989) reported a triphasic wave with one positive peak at $350 \mathrm{~ms}$ and two negative peaks at $200 \mathrm{~ms}$ and 1000 ms for salty stimulus of $\mathrm{NaCl}$ with a concentration of $10 \%$. They evaluated that the second negative peak was the response correlated to salty stimulus. Platting (1991) denoted that the influence of tactile sense to tongue could be removed with the use of a jet of distilled water by the application of the principle of olfactometer. He obtained the latency of $410 \mathrm{~ms}$ for negative peak and $1200 \mathrm{~ms}$ for positive peak for salty stimulus of $1.2 \mathrm{M} \mathrm{NaCl}$.

The results stated above showed the long latency of more than $200 \mathrm{~ms}$. The reason why the latencies obtained was longer than $200 \mathrm{~ms}$ and distributed at a wide range from 200 to 1500 ms depended on the apparatuses giving taste solution and detecting the moment that the taste solution touched to the tongue. The conventional apparatus used a long tube to give the taste solution. The apparatuses detected the moment (i.e., trigger timing) at the remote position from the tongue actually presented by 
the taste solution, so that the long latencies were measured. GEPs were correctly measured and systematically recorded by varying the concentrations of solutions of four taste qualities ( sweet-sucrose, salty-sodium chloride, sourtartaric acid, and bitter-quinine- $\mathrm{HCl}$ ) by averaging technique of evoked potentials and by detecting trigger time due to laser sensor (Min and Sakamoto, 1997 and 1998). In the previous papers (Min and Sakamoto, 1997 and 1998), the evoked potentials which averaged on eight consecutive responses for four taste qualities appeared positively with a latency at approximately $150 \mathrm{~ms}$. The amplitude and the latency of peak P2 at approximately 150 ms were influenced by the kind of taste qualities and the concentration of taste solution. GEP for sweet solution can be distinguished from somatosensory evoked potential by applying the sweet suppressing agent (Min and Sakamoto, 1998), while the effect of the sweet suppressing agent on other taste qualities except for sweet (i.e., salty, sour, and bitter tastes) was not recognized in the peak potential at the region of 150-200 ms. The artificial saliva and the solutions of four taste qualities produced the peak $\mathrm{P} 1$ at approximately $50 \mathrm{~ms}$. The result showed that the peak $\mathrm{P} 1$ generated from the origin without taste solution. Namely, the origin of P1 was considered to be somatosensory by touch of solution. The peak P1 was not affected by the treatment of a sweet suppressing agent. The results of the previous studies are related to the evoked potential due to stimulation of the tongue innervated by the chorda tympani and the whole oral cavity. Nothing has been reported on measurement of the evoked potential due to stimulation of the tongue innervated by the glossopharyngeal nerve. It is very difficult to stimulate the region of tongue innervated by glossopharyngeal nerve since the region exists at the interior of the tongue. This study was attempted to develop the measurement of the evoked potentials. In the electrical physiological experiment, there existed different sensitivity in the different regions of tongue for taste solutions. Especially, "Umami" taste solution, that enhanced flavor of food, showed different response of single nerve fibers for both the chorda tympani and the glossopharyngeal nerves of mouse by Ninomiya and Funakoshi (1987). They also found that some glossopharyngeal nerves were not activated by the taste solutions of sweet, salty, sour, and bitter substances, but they were activated strongly by Umami substances (e.g., MSG and guanylicmono phosphate (GMP) or a mixture of MSG and GMP) where Umami in J apanese language is designated as a pleasant gustatory sensation that is qualitatively different from the fundamental four taste qualities of sweet, salty, sour, and bitter. The evoked potential of Umami substances has not yet been measured. Here, we tried to measure the evoked potential stimulated by Umami substance MSG in the region of tongue innervated by the glossopharyngeal nerve.

\section{Materials and Methods}

\section{GEP apparatus and stimuli}

Stimulation solutions of four taste qualities (i.e., sweet, salty, sour, and bitter) for the region of tongue innervated by the chorda tympani nerve was taken using the apparatus developed for GEP recording ( $M$ in and Sakamoto, 1997). The apparatus for the gustatory stimulation of Umami for the region of tongue innervated by the glossopharyngeal nerve was improved as shown in Fig. 1. The experimenter generated the stimulus from the gustatory apparatus so that the taste solution moved from tastant bottle to tube. The site of stimulation was the tongue occupying foliate papilla innervated by the glossopharyngeal nerve. The speed of taste solution was introduced by the pressurized stimulator at the volume flow rate of $2 \mathrm{l} / \mathrm{min}$ adjusted by flowmeters ( Fig. 1). The volume of $1 \mathrm{ml}$ of tastant was given to the tongue. The bottle contained $20 \mathrm{ml}$ of the stimulating solution or the distilled water for a control. Solutions with some kinds of concentration were prepared. It is the mechanism of the detection that onset of gustatory stimulation is detected by the sensor with laser beam. The moment detected was used as trigger time. The trigger signal detected was sent from the device to electroencephalograph. The procedure for operating the stimulating solution is described as follows. The mouth was rinsed with distilled water in order to eliminate the effects of the saliva on the tongue. The tongue was protruded forward slightly, and the experimenter held the apex of the tongue with a piece of gauze. The experimenter inserted the tip of tube of the stimulating apparatus into the mouth of the subject with extreme care so as not to touch the apex of the tongue and oral cavity directly, and then pressed the switch of stimulating apparatus after making sure that EEG was stable. At this time, the evoked potentials were measured. At the end of each measurement, the subject was asked about the existence or absence of recognition of stimulating solution given, and if the solution was not recognized, the data were removed. An interval of 10 minutes was provided for the next test in order to eliminate adaptation and fatigue of the tongue by taste solution.

\section{GEP recording}

The subject was instructed to sit on a chair in a relaxed state. Potentials were recorded using a NeuroPack Eight Computer (Nihon-Kohden, Tokyo, Japan). The time constant of the amplifier was taken to be $0.3 \mathrm{sec}$ and the frequency of high-cut filter was set to be $30 \mathrm{~Hz}$. The recording position of the evoked potential was $\mathrm{Cz}$ and $A 1$ which was taken on the left lobe as reference position, and the ground was set on the Fpz. The silversilver chloride $(\mathrm{Ag} / \mathrm{AgCl})$ electrodes with $7 \mathrm{~mm}$ in 
Device of laser beam

(Trigger signal is sent to EEG apparatus.

The inner diameter of sensor is $2 \mathrm{~mm}$ ).

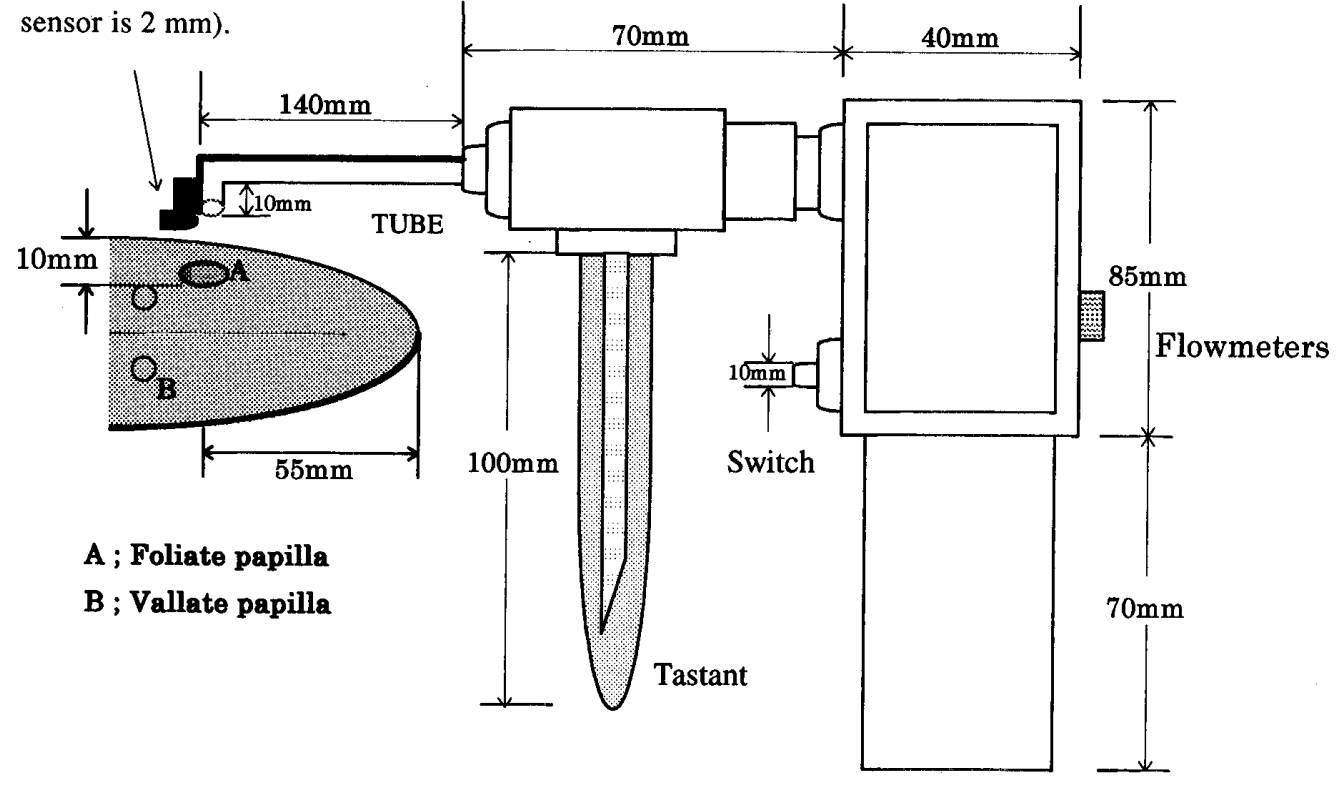

Fig. 1 The schematic depiction of apparatus for gustatory stimulus supply to tongue. The shape of unit for supplying solution is tube length $140 \mathrm{~mm}$ and inner side $3 \mathrm{~mm}$. When the solution passes to the tip of the tube, the sensor with laser beam detects the transfer of the solution. The moment detected was used as trigger time.

diameter (Nihon Kohden, NE-121B) were used. The stimulation of the taste solution was performed by eight times and the waveform of each evoked potential was recorded, and after the measurement they were averaged.

Kobal (1985) fixed 50-60s for the time intervals of stimulations with a taste solution. Here, in order to avoid adaptation when stimuli were repeatedly presented, the interval was taken to be $60 \mathrm{~s}$ and the duration of stimulus was taken to be $200 \mathrm{~ms}$. The temperature of both all taste solutions and the control solution (i.e., distilled water) was $35^{\circ} \mathrm{C}$ (equal to skin temperature) to eliminate the effects of warm sensation. The interelectrode resistance was adjusted to be less than $5 \mathrm{k} \Omega$. The analysis time was $1000 \mathrm{~ms}$. Ten healthy male subjects who were of age 24 to 27 years and non-smokers tried to receive the stimulus of taste solution to the region innervated by the glossopharyngeal nerve. The efficiency of the new apparatus by the stimulation of taste solution was evaluated. The environmental temperature of the test room was maintained between 20 to $23^{\circ} \mathrm{C}$ and the time period for the measurement was taken to be 2:00 to 5:00 in the afternoon. All the conditions of the measurement were the same as the previous paper (Min and Sakamoto, 1998).

\section{Results and Discussion}

\section{GEP recording}

The apparatus which stimulated chorda tympani nerve by taste solution (Min and Sakamoto, 1998) could not give the stimulus to the interior part of tongue, that is, the region innervated by glossopharyngeal nerve, because the tip part of the apparatus giving the taste solution was too large for the interior part of tongue. But the new apparatus used in this study i.e., the part giving the taste solutions to the tongue was concentrated on a small area as shown in Fig. 1. The distance between the tip part and the surface of the region of tongue innervated by glossopharyngeal nerve was operated by the experimenter. The passage of the taste solution to the mouth of tube in the apparatus was detected by a sensor with laser beam. The correct touching moment of taste solution to the tongue need to be acquired. Owing to the improvement of the conventional apparatus (Min and Sakamoto, 1998), the evoked potentials by stimulus of solution of MSG to glossopharyngeal nerve could be able to be measured in the study for the first time. However, the difficulty to detect the response for distilled water as the control existed due to small amplitude of the peak (Fig. 2). The evoked potential of Umami has not yet been reported, 


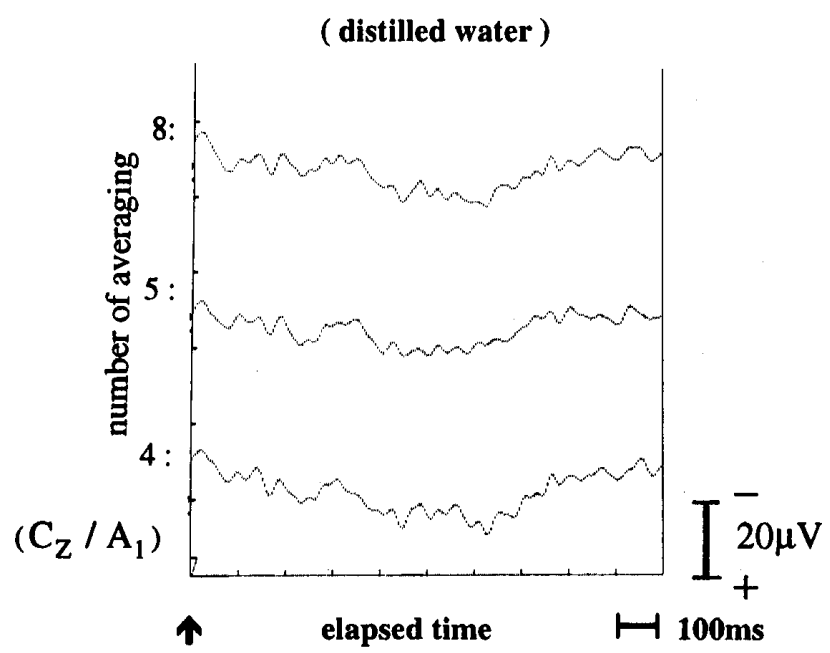

Fig. 2 Evoked potentials for the subject who denoted the average amplitude of N1-P2 by distilled water as the control. The arrow indicates the onset of stimulation.

because the main region activated by Umami substance was known to be the most interior part of tongue innervated by glossopharyngeal nerve (Yamaguchi, 1997; Ninomiya and Funakoshi, 1987). The new apparatus which could measure the evoked potential for Umami solution materialized. In the study the evoked potential for MSG solutions as Umami substance was treated. The typical pattern of an evoked response of 0.05 M MSG before averaging are shown in the upper part of Fig. 3, and the lower part is eight times the average of the evoked potentials which showed the main positive peak at approximately $250 \mathrm{~ms}$. The optimal number of average of evoked potentials for MSG was investigated. The positive response was able to be detected at a certain peak latency by the average (Fig. 4). In the graph shown, the number 8 represents the average of eight waves (i.e., eight evoked potentials). The average of more than four waves showed clear similar latency. The average of the large number of waves which showed more than ten times denote the lower positive peak for the measured time until $1000 \mathrm{~ms}$. The phenomenon was due to the adaptation of the taste sensation on the tongue. In the measurement of the evoked potentials for the region innervated glossopharyngeal nerve, the maximum positive peak could be obtained in the average number of less than 10 times. The results of all the subjects denoted that the average of eight waves gave clear peak potential and the latency. Here, the average of eight waves was treated to evaluate the latency. If the rest period was short by less than $10 \mathrm{~min}$, the evoked response was unclear in the following test. The values of the mean $(\bar{x})$ and the standard deviation (SD) of the evoked potential of MSG gave a peak latency at $248 \pm 15 \mathrm{~ms}$ for all the subjects. As for the amplitude of the evoked potentials, the potential difference between the peak

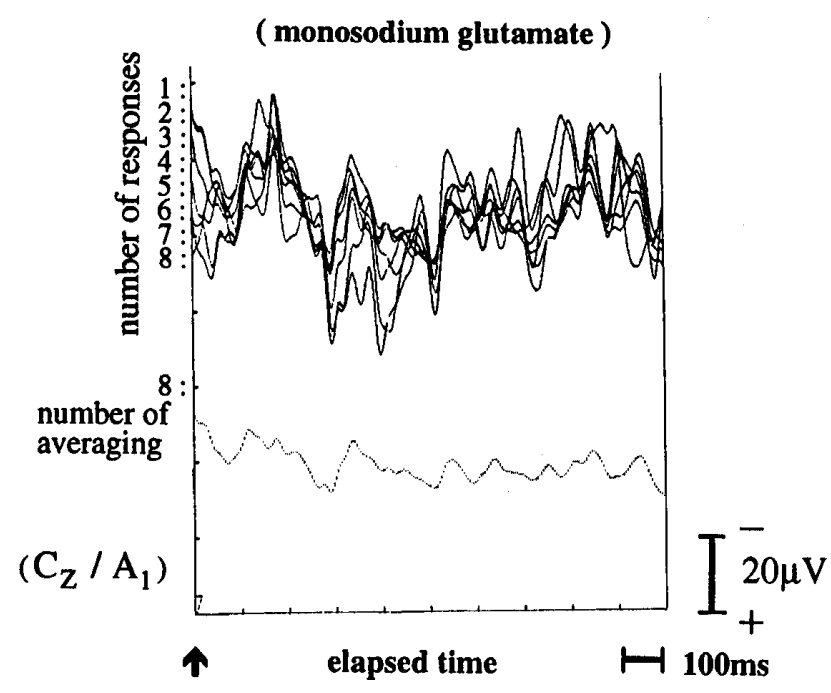

Fig. 3 Typical pattern of evoked potentials by gustatory stimulations of $0.05 \mathrm{M}$ monosodium glutamate (MSG). The evoked potentials show good reproducibility. Before the averaging, the positive responses were detectable at a certain peak latency by the superimposition of eight evoked potentials at lower part. The arrow indicates the onset of stimulation. As for the subject, the same subject as in Fig. 2.

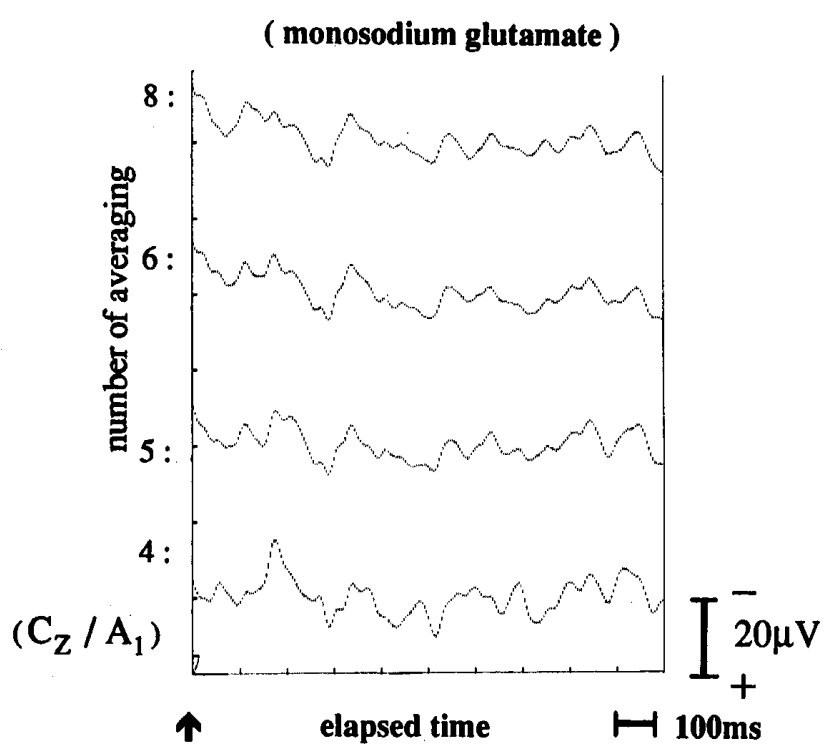

Fig. 4 Averaged evoked potentials of $0.05 \mathrm{M}$ monosodium glutamate (MSG). The positive wave evoked by the tastant was obvious with averaging. The arrow indicates the onset of stimulation. As for the subject, the same subject as in Fig. 2.

potential prior to $\mathrm{P} 2$ which was called $\mathrm{N} 1$ and the peak potential of $\mathrm{P} 2$, that is N1-P2, was obtained from the averaged wave of evoked potential. The values of $\bar{x}$ and SD of N1-P2 for the taste concentrations of MSG with $0.05 \mathrm{M}$ and $0.1 \mathrm{M}$ denoted $16 \pm 3 \mu \mathrm{V}$ and $27 \pm 7 \mu \mathrm{V}$, respectively. 


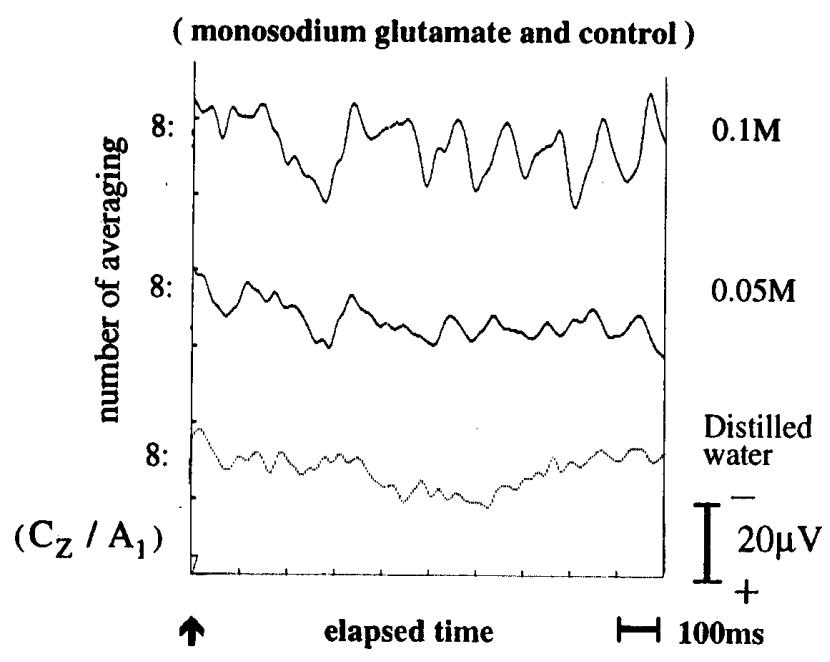

Fig. 5 The average evoked potentials for two different concentrations of MSG and the potential for distilled water. The arrow indicates the onset of stimulation. As for the subject, the same subject as in Fig. 2.

On the other hand, as for the distilled water, the values of the potential difference between the potential generated the time corresponding to $\mathrm{N} 1$ and the potential of $\mathrm{P} 2$ gave $4 \pm 3 \mu \mathrm{V}$. The statistical significant difference between the potential difference N1-P2 of MSG and the peak of the distilled water at the same period was obtained at the level of $1 \%$. Moreover, in order to evaluate the influence of the concentrations on evoked potentials, two kinds of the concentration of MSG were used. Fig. 5 shows the evoked response for two concentrations of $0.05 \mathrm{M}$ and 0.1 $M$ of MSG and the response for the distilled water. The most prominent component of the evoked potentials denoted a positive peak occurring at approximately 250 ms. It was found that the peak latencies decreased and the amplitudes increased as the concentration of MSG increased and that the peak response of MSG was able to be discriminated from that of control solution (i.e., distilled water).

Acknowledgment. The author is indebted to Prof. Dr. Yamaguchi S. of the Tokyo University of Agriculture, who supplied the monosodium glutamate.

\section{References}

Chiappa KH (1983) Evoked potentials in clinical medicine. Raven Press, New York, 26-270

Funakoshi M, Kawamura Y (1968) Summated cortical responses to taste stimulation in man. J Physiol Soc J pn 30: 282-283

Funakoshi M, Kawamura Y (1971) Summated cerebral evoked responses to taste stimuli in man. E lectroenceph clin Neurophysiol 30: 205-209

Ikui A (1988) Gustatory evoked potentials induced by stimulation with $\mathrm{NaCl}$ solution in man. J Nihon Univ Med Ass 47 (3) : 363-373

Kobal G (1985) Gustatory evoked potential in man. Electroenceph clin Neurophysiol 62: 449-454

Maetani C, Notake N, Takemoto I, Koizuka I, Ogino H, Matsunaga T, Yoshimura S, Tonoike M (1989) Gustatory evoked potentials by taste solution. Chem Senses 14 (2): 311

Min BC, Sakamoto K (1997) An analysis of gustatory evoked potentials for the four qualities with a special reference to individual taste thresholds. J pn J Taste Smell Res 4 (1): 43-54

Min BC, Sakamoto K ( 1998) Influence of sweet suppressing agent on gustatory brain evoked potentials generated by taste stimuli. Appl Human Sci 17(1): 9-17

Murayama N, Nakasato N, Hatanaka K, F ujita S, Igasaki T, Kanno A, Yoshimoto T (1996) Gustatory evoked magnetic fields in humans. Neurosci Lett 210: 155-158 Ninomiya Y, Funakoshi M (1987) Qualitative discrimination among umami and the four basic taste substances in mice. In Kawamura $Y$, Kare MR eds. Umami-A basic taste. Marcel Dekker Inc. New York, 365-385

Platting KH (1991) Gustatory evoked brain potentials in humans. In Getchell TV, Bartoshuk LM, Doty RL, Snow JB eds. Smell and taste in health and disease. Raven Press, New York, 277-286

Yamaguchi S (1997) Umami. In Sato M, Ogawa H eds. Mikakunokagaku. A sakura Publish Inc. Tokyo, 25-35

Received: September 23, 1997

Accepted: J anuary 29, 1998

Correspondence to: Kazuyoshi Sakamoto, Ergonomics Lab., Department of Communications and Systems, The University of E lectro-Communications, 1-5-1 Chofugaoka, Chofu City, Tokyo 182, Japan 